\title{
Development of Durability Test Procedure of Vibration- Based Energy Harvester in Railway Vehicle
}

\author{
Ji-Won Jin', Ki-Weon Kang',, and Jae-Hoon Kim² \\ 1 School of Mechanical and Automotive Engineering, Kunsan National University, 558, Daehak-ro, Gunsan-si, Jeollabuk-do, 54150, South Korea \\ 2 High Speed Railroad System Research Center, Korea Railroad Research Institute, 176, Cheoldobangmulgwan-ro, Uiwang-si, Gyeonggi-do, 16105, South Korea \\ \# Corresponding Author / E-mail: kwkang68@kunsan.ac.kr, TEL: +82-63-469-4872, FAX: +82-63-469-4727
}

KEYWORDS : Durability test procedure, Durability test system, Power spectral density (PSD), Vibration-Based energy harvester

This study aims to develop a durability test procedure for vibration-based energy harvesters in the frequency domain. First, we investigated the vibration environment of a structure on which an energy harvester may be installed and determined the level and frequency range of the power spectral density (PSD) exerted on the energy harvester. Consequently, we developed a test procedure, which is composed of the PSD and sinusoidal sweep loads. The test system was built using a vibration exciter, data acquisition unit, and LabView-based control software. Using the developed test system, durability performance tests for two types of energy harvesters were performed according to the established test procedure.

\section{Introduction}

In recent years, the continued drop in power requirement for electronic sensors ${ }^{1}$ has led to remarkable scientific and engineering interest in energy harvesting technologies. ${ }^{2}$ Energy harvesters can use various energy sources such as electromagnetic, photovoltaic, vibrational, thermal, hydroelectric, and wind power. ${ }^{3}$ Among them, mechanical vibration is one of the most promising energy sources that could be exploited for many applications in their relevant environments.

Vibration-based energy harvesters (VEHs) ${ }^{4}$ utilize the vibration generated from various structures and/or components and specifically, are designed that their natural frequencies are within the frequency range of dynamic loads that originate from the structures and/or components and consequently augment the displacement of moving parts in VEH, in order to enhance the power production efficiency. ${ }^{5}$ Such VEHs intend to replace or extend service life of batteries for condition monitoring system for high-risk structures such as railway vehicle and wind turbine, in which it is hard to supply the electrical power for data measurement and transmission. ${ }^{6}$ The condition monitoring system should measure and transfer the safety-related data of high-risk structures during their service life without intermission. Hence, VEHs should have the sufficient durability, to monitor the safety or structural integrity of high-risk structures. Therefore, it is more important than ever to evaluate the durability of $\mathrm{VEH}$ in order to make VEHs reliable for monitoring the condition of high-risk structures.

The durability of structures under an irregular load is usually evaluated under random loads, expressed in a power spectral density (PSD) form of the exciting forces such as accelerations. There are some standards and researches on durability evaluation for the structures/ components under random loads. ${ }^{7-10}$ IEC $61373^{7}$ standard encompasses the requirements for random vibration and shock testing of various equipment to be installed in railway vehicles. Particularly, this standard specifies the accelerated life test methodology of the so-called "simulated long life test," which is aimed at establishing the mechanical integrity of equipment at increased service levels. SAE $\mathrm{J} 1455^{8}$ standard describes various environments exhibiting mechanical vibration; these are environments that influence the performance and reliability of electronic equipment designed for heavy-duty on- and offroad vehicles. Furthermore, $\mathrm{Su}^{9}$ presented an engineering procedure to determine a justified vibration load specification for design validation tests of automotive products based on measured vehicle load data. This procedure is presented in either sinusoidal sweep or random vibration formats in the frequency domain, both of which have equivalent durability damage levels for the given test duration and reliability parameters. Chen et al. ${ }^{10}$ developed an accelerated random vibration test for the joint reliability of cantilevered compressor shell 
attachments. Their method provided an accelerated random vibration fatigue test including the test spectrum, test duration, and failure criteria. As discussed so far, various results have been presented to evaluate the durability of structures and/or components under random vibration loads. However, although the structures and components in the aforementioned standards and researches are under random vibration loads, they are designed to avoid resonance with the excitation frequency of the random vibration exciting loads. Meanwhile, VEHs are designed to actively exploit the resonance between the excitation frequency and the natural frequency of VEHs. Additionally, while the aforementioned references mostly define malfunction or structural damage as failure criteria, such failure criteria cannot be applicable to VEHs, because their intrinsic function is to generate power output above certain power level. Therefore, the functional failure criterion must be the case in which the power output falls below a predefined threshold level. However, such a failure definition has not been clearly established.

The goal of this paper is to propose a procedure to evaluate the durability of a vibration-based energy harvester under random vibration loads. For this purpose, we first define the intrinsic function of VEHs and then set up the measurement scheme in the frequency domain. Furthermore, based on our measurement scheme, we define a durability test procedure which can measure and evaluate performance degradation and durability under random vibration loads expressed in PSD. Finally, we build a durability test system for VEHs according to our durability test procedure and carry out the durability tests for two types of cantilevered energy harvesters.

\section{Durability Test Procedure for Energy Harvester}

\subsection{Vibration-Based Energy Harvester}

The VEHs studied in this paper are cantilevered electromagnetic energy harvesters, whose mechanism are shown in Fig. 1. These VEHs are designed to have a natural frequency of about $51 \mathrm{~Hz}$ to resonate with the excitation frequency of random vibration loads originated from high-risk structures. Also, the VEHs generate a maximum power of about $10 \mathrm{~mW}$ at $1 \mathrm{~g}\left(\mathrm{~g}=9.81 \mathrm{~m} / \mathrm{s}^{2}\right)$ and corresponding natural frequency.

\subsection{Test Procedure for the Vibration-Based Energy Harvester \\ 2.2.1 Test for Reference Performance}

The cantilevered VEHs studied in this paper are system that swells power output using the resonance between the natural frequency of the VEH and the excitation frequency of random vibration from the highrisk structure. ${ }^{11}$ Since the power output from the VEHs depends on the excitation frequency of random vibration as well as the natural frequency of the VEHs, it is reasonable to define the peak of power output at the resonance frequency of the VEHs as the rated power of the VEHs.

Considering the intrinsic function of VEHs, we define the reference performance of the VEHs as the peak of power output measured when the frequency of an excitation load is the same as the natural frequency of the VEHs. As experimental conditions, we set the amplitude and frequency range as $1 \mathrm{~g}$ and [the natural frequency of VEHs] $5 \mathrm{~Hz}$, respectively, in a sinusoidal sweep test, which was applied to evaluate the reference performance of the VEHs, in order to overcome uncertainties in the evaluation of natural frequency of the VEH and the accuracy of the vibration exciter. We also set the peak value from the power output time series under the sinusoidal sweep test as the reference performance value or reference power. Fig. 2 shows the reference performance test condition defined so far.

\subsubsection{Durability Block Test for Energy Harvester}

VEHs are based on a mechanism that generates power using the resonance between the excitation frequency of random loads from high-risk structures and the natural frequency of the VEHs. This random load is an energy source for the VEHs, but it produces fatigue damage at the same time. Fatigue damage in VEHs can cause a change in stiffness, consequently changing the frequency response of the corresponding structures. Thus, with a change in the natural frequency of a VEH due to accumulated fatigue damage, its power output may
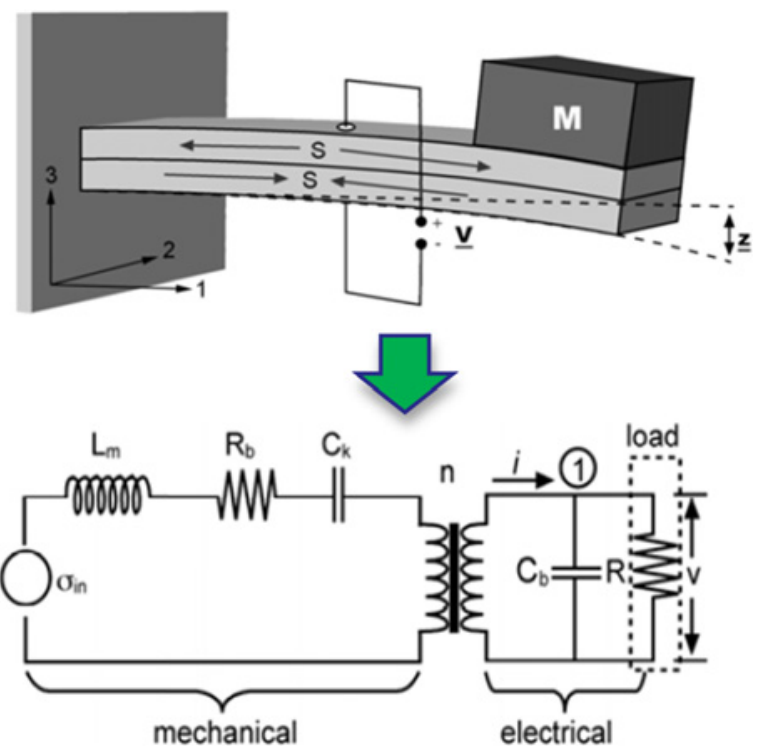

Fig. 1 Schematic diagram of cantilevered electromagnetic energy harvester

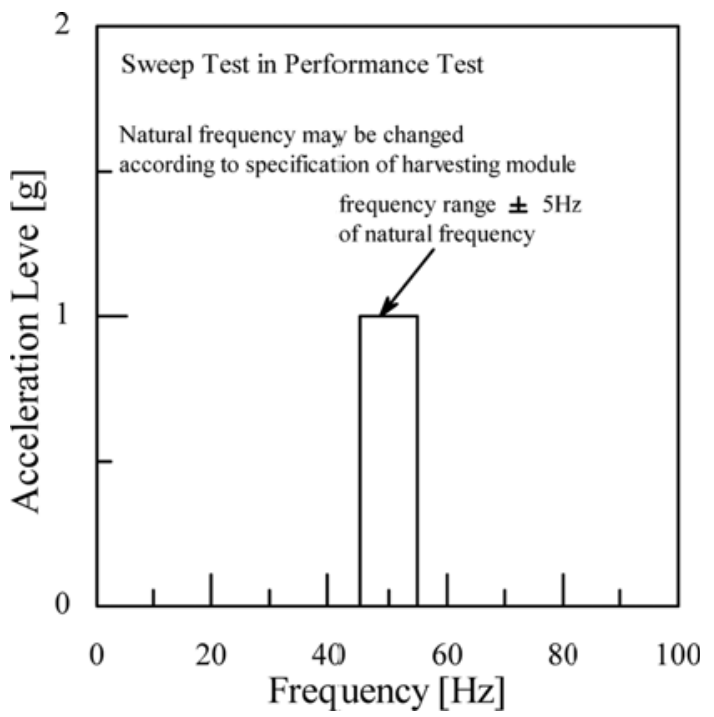

Fig. 2 Test condition for the reference performance of energy harvester 
decrease. Therefore, to evaluate the durability of VEHs, we should measure the change in the power output under a random load, which is the energy source of the VEH as well as the cause of fatigue damage. Based on such concepts, we define a block test to evaluate the durability of a VEH as follows.

First, a random load that originates from corresponding high-risk structures is applied to VEHs according to a predefined test duration. Fig. 3 shows a typical time series of a random load from a high-risk structure. Because the vibration loads from the structures such as railway vehicles and wind turbines are random in nature, they are generally defined by PSD forms in the frequency domain. ${ }^{7}$ Thus, we also define the random load using PSD in this work; the PSD level and frequency range are indispensable in defining random loads in a PSD form. We choose the PSD level and frequency range based on IEC $61373,{ }^{7}$ which is for a railway vehicle, one of the representative highrisk structures. Note that the PSD level and frequency range can be updated once the measurement and analysis of the vibration environments of the corresponding high-risk structures are completed.

Second, we need a standard evaluation method to measure the reduction behavior of power output under random loads due to the

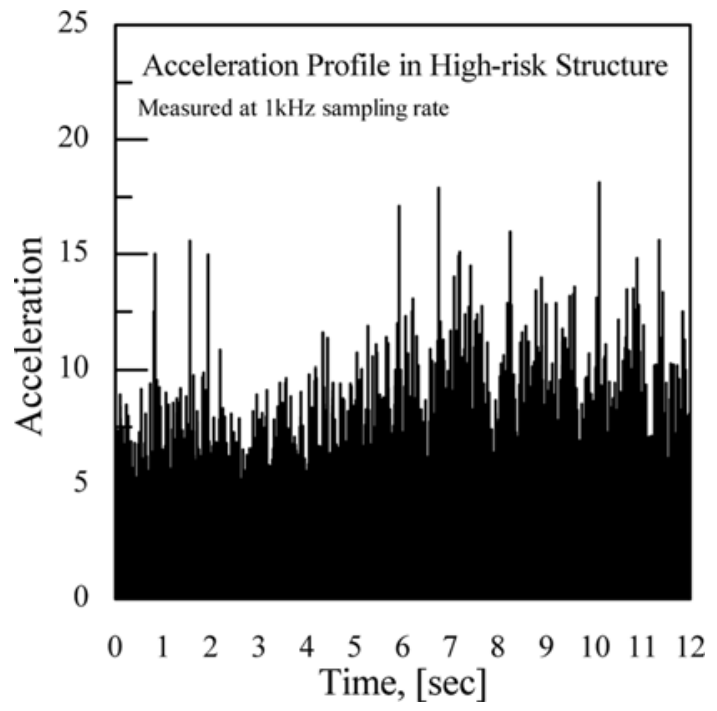

Fig. 3 Typical random load time series measured at high-risk structure
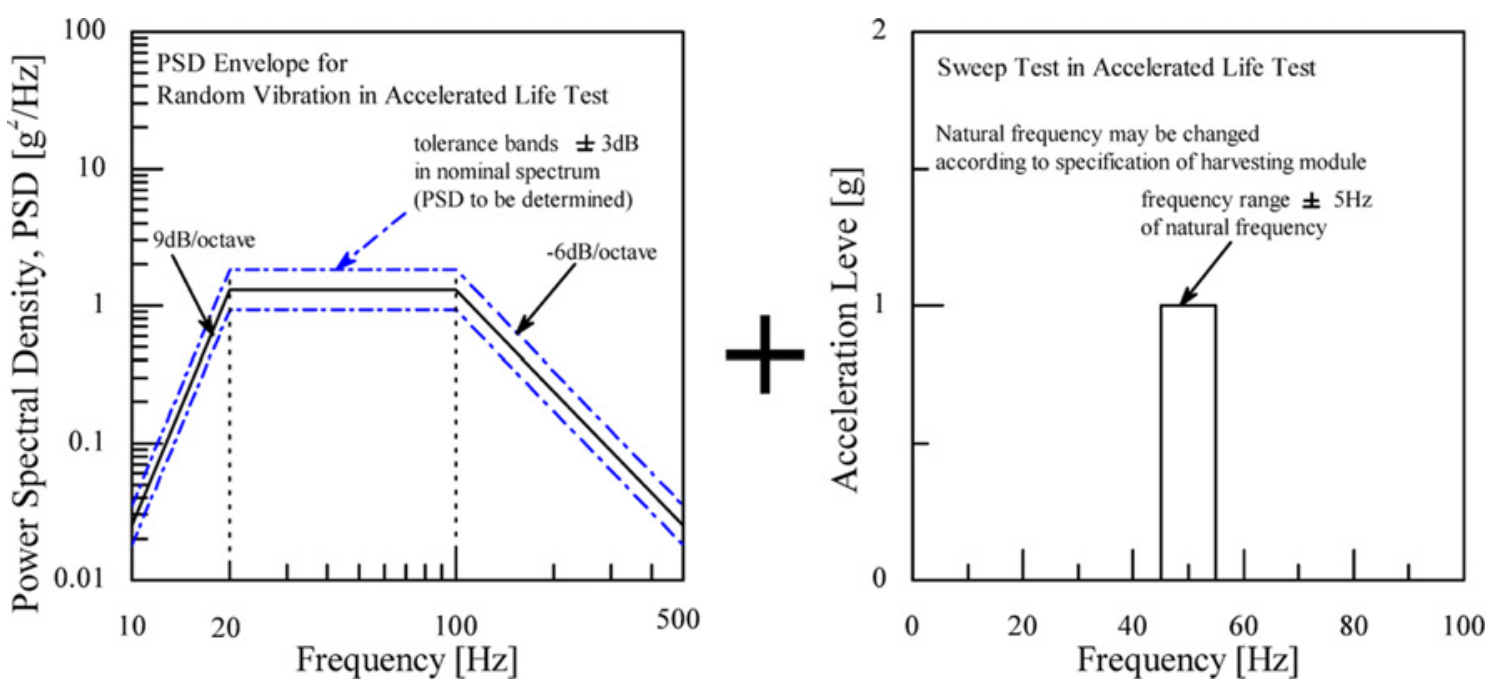

fatigue damage and resulting the change in the natural frequency of the VEHs. Comparing the power output under random loads with reference performance of the VEHs leads us to identify the degradation characteristics of the VEHs. Therefore, we set the measurement scheme under a durability block test identical to the reference performance measurement in Section 2.2.1, in which the measured power output is called the block power output. Fig. 4 shows the schematic diagram of proposed durability block test for VEHs.

\subsubsection{Failure Criteria for Energy Harvester}

As previously mentioned, VEHs generate power and suffers from accumulated fatigue damage at the same time, changing the stiffness and natural frequency and resulting in reduced power output. From this perspective, we can define two types of failures with respect to the durability of VEHs: functional failure in which power output degrades below a certain threshold losing the intrinsic function of VEHs and structural failure in which no power is generated owing to the completely fractured core components. Since structural failure indicates that no power is generated, its definition is very straightforward. However, functional failure means that the VEHs still generate power,

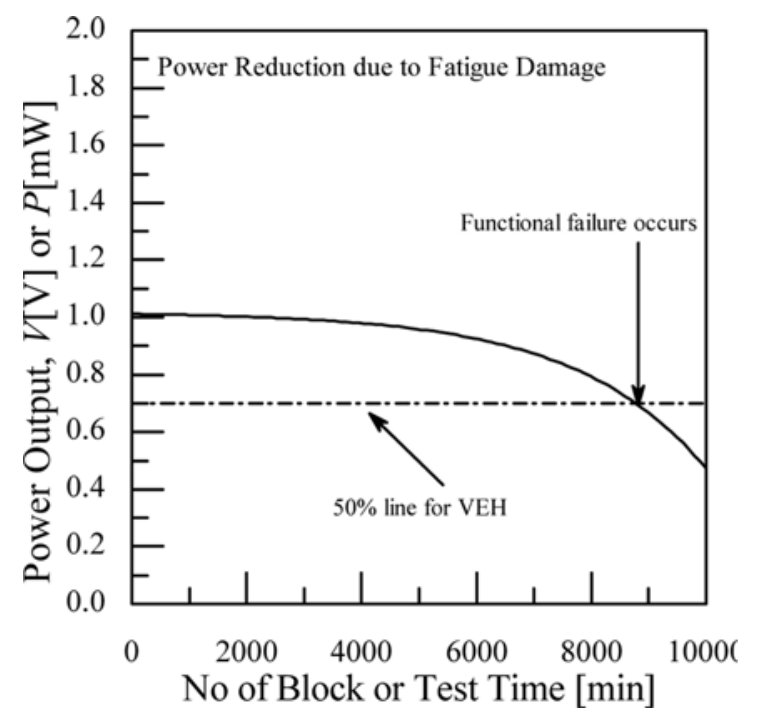

Fig. 5 Schematic diagram of power reduction of VEH against blocks

Fig. 4 Definition of a durability block 
but it is below a certain level. Accordingly, we should set the threshold value of functional failure as the minimum power for a condition monitoring system in which the VEHs are installed. Since there is no clear specification on such threshold value, we define functional failure as a case in which the output is $50 \%$ below the reference performance of VEHs. The service life for the functional failure of VEHs (henceforth called the 'functional life') is the number of durability blocks repeated until the power output degrades by $50 \%$, as shown in Fig. 5 .

\subsection{Procedure of Durability Test for Vibration-Based Energy Harvester}

Fig. 6 summarizes the experimental method explained in Section 2.2, which leads to the following test procedure.

(1) Reference performance test (sinusoidal sweep only) will be performed to measure the reference power in which the frequency range and acceleration level are initially set to [the natural frequency] $\pm 5 \mathrm{~Hz}$ and $1 \mathrm{~g}$, respectively, although they may change according to the characteristics of the VEHs. We measure the power using a data acquisition unit (DAU) and set the peak power as the reference power.

(2) Once the reference performance is established, the VEH is subjected to block (PSD and sinusoidal sweep) tests in which the power is measured only in the sinusoidal sweep test. The obtained peak power is called the block power output in the corresponding block.

(3) The measured block power output is compared with the reference power, and if it is higher than a predefined threshold $(50 \%$ with respect to the reference power), the test in (2) is repeated. Otherwise, the service life at this moment is defined as the functional or structural failure life.

(4) In case the functional and structural failures occur sequentially because of the characteristics of the VEHs, the functional or structural life is determined according to whether the block power output exits. If the VEH generates still power output, the life means the functional life. Otherwise, it is the structural life.

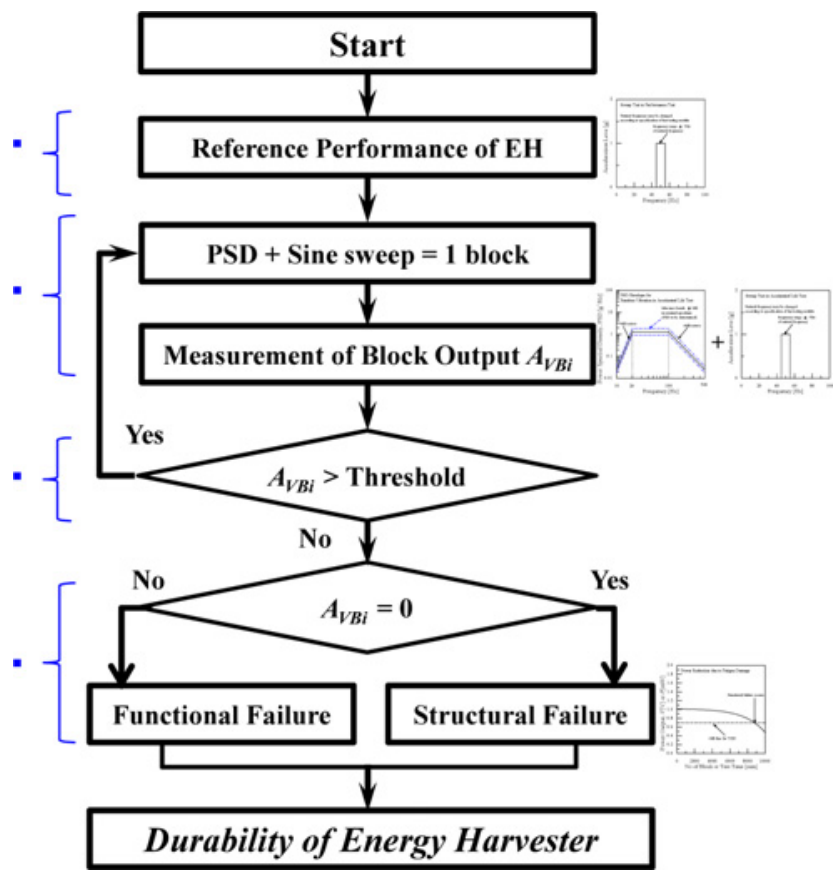

Fig. 6 Flowchart of durability test for vibration-based energy harvester

\section{Test System Buildup and Test Results}

\subsection{Test System Buildup}

Fig. 7 shows the durability test system configured for the test procedure in Section 2, the details of which are explained in the following.

\subsubsection{Random Vibration Exciter}

The random vibration exciter used to apply the PSD and sinusoidal sweep load to the VEHs is an electro-magnetic vibration shaker (FAMTECH, EDS150-150). This vibration shaker has a useable frequency range of $5-4,500 \mathrm{~Hz}$ and a maximum displacement of $25 \mathrm{~mm}$. This exciter is controlled by dedicated software that sets up test loads, starts and stops the equipment, and sends event signals.

\subsubsection{Data Acquisition Unit for Power Measurement of VEHs}

Measuring the reference and block power output described in Section 2 is essential to evaluate the durability of VEHs. In the present study we use a DAU (National Instruments) to measure the power output in terms of voltage during the sinusoidal sweep test. The analog voltage signal measured by this DAU is converted into a digital signal at a sampling rate of $1 \mathrm{kHz}$. We then transmitted the digital signal to a dedicated software, as described in Section 3.1.3.

\subsubsection{Energy Harvester Power Monitoring Software}

We need to analyze the reference and block power outputs measured by the hardware in Sections 3.1.1 and 3.1.2 to evaluate the durability of VEHs; this is accomplished by a LabView-based energy harvester power monitoring (EHPM) software. Our software finds the peak values from a power (voltage) time series measured during the reference performance test and then sets it as the reference power. Furthermore, it measures the block power output during the block test. If the measured value is larger than a failure threshold (50\%) after being compared with the reference power, the software commands the vibration shaker to continue the block test. Otherwise, it commands the vibration shaker to stop the test and store all the data collected until that moment.

\subsection{Durability Performance Test Results}

To verify the effectiveness of test procedure and test system

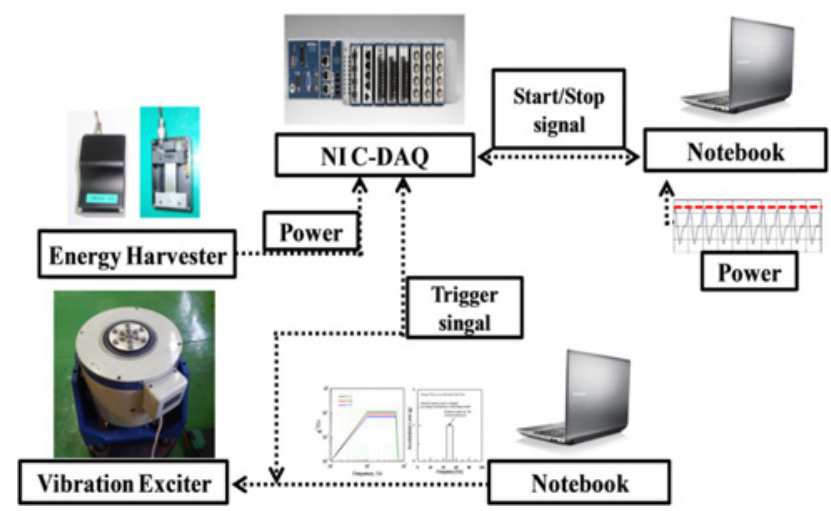

Fig. 7 Configuration of durability test system for vibration-based energy harvester 
discussed so far, we performed durability tests on two types (Type I and Type II) of cantilevered vibration-based energy harvesters. Here, Type I and II VEHs have the same structural design but different beam materials. Hence, they have same natural frequencies but different damping coefficients. The applied PSD level and duration of the tests were set as 0.02 and $3600 \mathrm{~s}$, respectively. Furthermore, the frequency range and level for the sinusoidal sweep test were $51 \pm 5 \mathrm{~Hz}$ and $1 \mathrm{~g}$, respectively. Under these conditions, we performed the test on Type I and Type II VEHs in which there were 10 blocks in total.

We stored the voltage time history for each block test, and Fig. 8 shows the typical results. Regarding the result for Type I, the voltage reached the maximum value at about $4.3 \mathrm{~s}$. Also sharply dropped values at before and after maximum value indicate that the natural frequency of the VEH was reached. Furthermore, we found a second peak around $9.8 \mathrm{sec}$, which corresponds the case in which the frequency drops from a upper bound $(56 \mathrm{~Hz})$ and eventually reaches the natural frequency, considering the sequence in the sinusoidal sweep

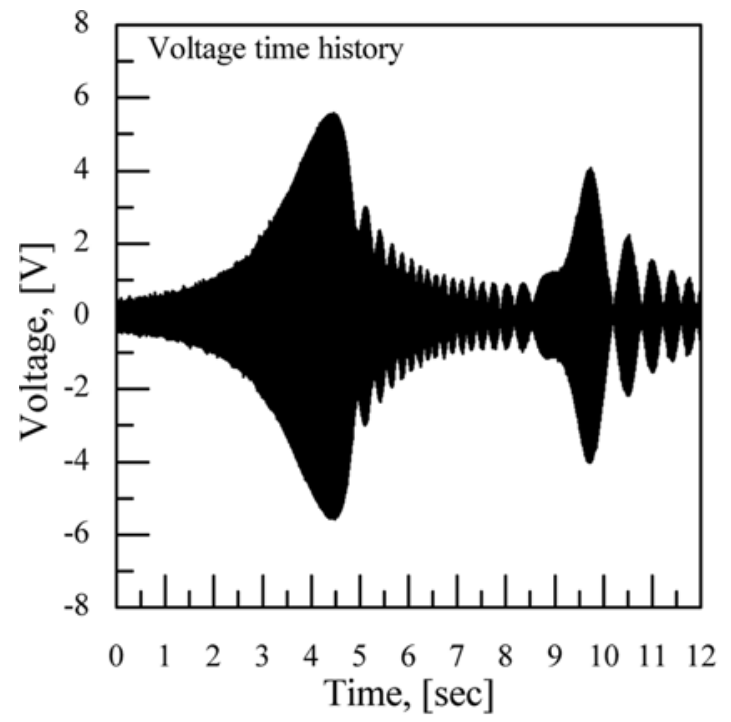

(a) Type I

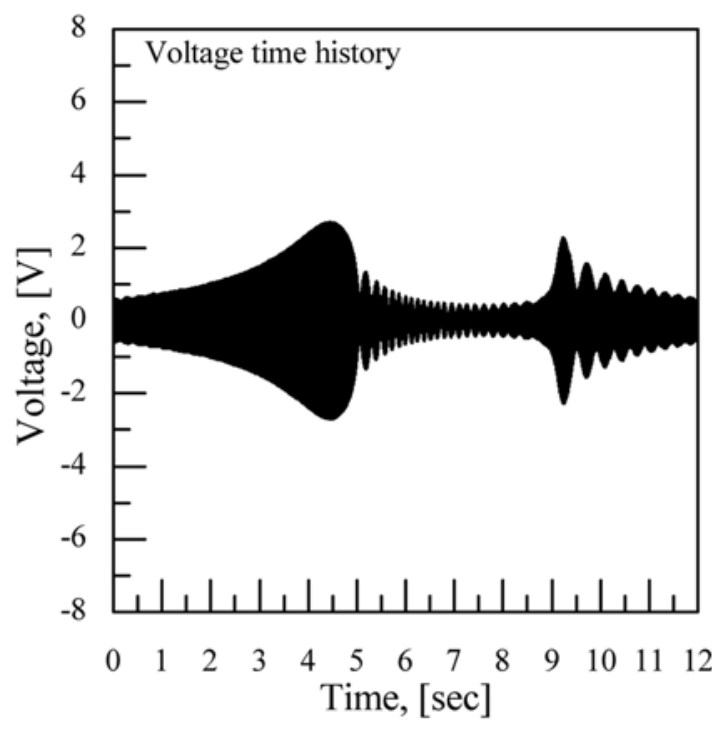

(b) Type II

Fig. 8 Voltage time history of VEH at $5^{\text {th }}$ block test (46 Hz natural frequency of the VEH (first peak) $56 \mathrm{~Hz}$ natural frequency (second peak) $46 \mathrm{~Hz}$ ). Here, it is thought considered the reason why the peaks are different even with the same natural frequency is the inertia effect of the cantilevered beam and attached mass. The result for Type II, the core components of which are different from those in Type I, showed very similar behavior except for minor differences in voltage rising characteristics due to different material characteristics. From our results, we conclude that it is feasible to use a sinusoidal sweep including the natural frequency to measure the reference and block power from VEHs considered herein.

Fig. 9 plots the peaks from the voltage time histories over block numbers. We found negligible change in block power(voltage) output from the VEH regardless of the type. This behavior is thought to come from the insufficient fatigue damage due to too low PSD level $\left(g_{\mathrm{rms}}=2.7 \mathrm{~g}\right)$ and too short test duration (10 blocks). In further works, we will apply the higher random vibration load to various VEHs until they would break.

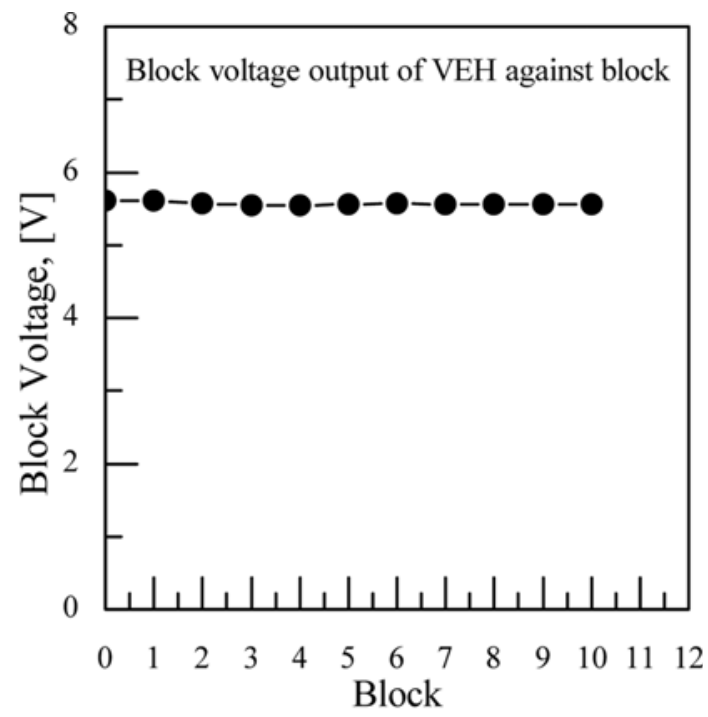

(a) Type I

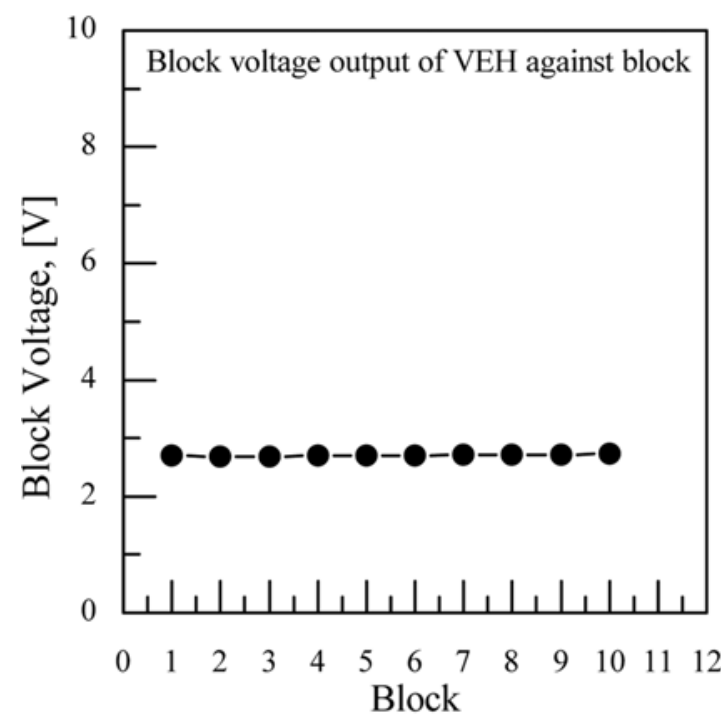

(b) Type II

Fig. 9 Block voltage output of VEH against block 


\section{Conclusions}

In this paper, we proposed a methodology to evaluate the durability of a vibration-based energy harvester under a random vibration load. Our results are as follows:

(1) We defined the basic features of VEHs and then proposed a durability test procedure which can evaluate the performance degradation and durability of VEHs under random vibration loads expressed in terms of PSD.

(2) We constructed the durability test system based on the proposed durability test procedure for VEHs and applied it to two types of cantilevered energy harvesters.

\section{ACKNOWLEDGEMENT}

This work was financially supported by the Human Resources Development of the Korea Institute of Energy Technology Evaluation and Planning (KETEP) through a grant funded by the Korean government's Ministry of Knowledge Economy (No. 20144030200590) and by the R\&D Convergence Program of MSIP (Ministry of Science, ICT and Future Planning) and NST (National Research Council of Science \& Technology) of Republic of Korea (Grant B551179-12-06-00).

\section{REFERENCES}

1. Moss, S. D., McLeod, J. E., Powlesland, I. G., and Galea, S. C., "A Bi-Axial Magnetoelectric Vibration Energy Harvester," Sensors and Actuators A: Physical, Vol. 175, pp. 165-168, 2012.

2. Priya, S. and Inman, D. J., "Energy Harvesting Technologies," Springer, 2009.

3. Andosca, R., McDonald, T. G., Genova, V., Rosenberg, S., Keating, J., et al., "Experimental and Theoretical Studies on MEMS Piezoelectric Vibrational Energy Harvesters with Mass Loading," Sensors and Actuators A: Physical, Vol. 178, pp. 7687, 2012.

4. Roundy, S., "On the Effectiveness of Vibration-Based Energy Harvesting," Journal of Intelligent Material Systems and Structures, Vol. 16, No. 10, pp. 809-823, 2005.

5. Goldschmidtboeing, F. and Woias, P., "Characterization of Different Beam Shapes for Piezoelectric Energy Harvesting," Journal of Micromechanics and Microengineering, Vol. 18, No. 10, Paper No. 104013, 2008.

6. Roundy, S., Wright, P. K., and Rabaey, J., "Energy Scavenging for Wireless Sensor Networks with Special Focus on Vibrations," Kluwer Academic Publishers, 2004.

7. IEC No. 61373, "Railway Applications - Rolling Stock Equipment Shock and Vibration Tests," 2011.

8. SAE No. J1455, "Recommended Environmental Practices for Electronic Equipment Design in Heavy-Duty Vehicle Applications," 2006.
9. Su, H., "Vibration Test Specification for Automotive Products Based on Measured Vehicle Load Data," SAE Technical Paper, Paper No. 2006-01-0729, pp. 1-13, 2006.

10. Chen, J. and Draper, D., "Random Vibration Fatigue Tests to Prove Integrity of Cantilevered Attachments on Compressor Shells," Proc. of the International Compressor Engineering Conference, pp.1570$1577,2002$.

11. Mak, K. H., McWilliam, S., Popov, A. A., and Fox, C. H. J., "Performance of a Cantilever Piezoelectric Energy Harvester Impacting a Bump Stop," Journal of Sound and Vibration, Vol. 330, No. 25, pp. 6184-6202, 2011. 